\title{
Ant Lion Optimizer based Approach for Optimal Scheduling of Thermal Units for Small Scale Electrical Economic Power Dispatch Problem
}

\author{
Navpreet Singh Tung ${ }^{1}$, Sandeep Chakravorty ${ }^{2}$ \\ ${ }^{1}$ Assistant Professor, Department of Electrical Engineering, \\ Bhutta Group of Institutions, Ludhiana, India \\ ${ }^{2}$ Dean and Professor, Department of Electrical Engineering, \\ Baddi University, Baddi, India \\ ${ }^{1}$ icenitj@gmail.com \\ ${ }^{2}$ sandeep@baddiuniv.ac.in
}

\begin{abstract}
A novel nature inspired algorithm ant lion optimizer (ALO) is recently developed which is motivated from the hunting mechanism of ant lions. Inherit steps of hunting prey such as the random walk of ants, building traps, entrapment of ants in traps, catching preys, and re-building are simulated to find the optimal solution of real life problems . Intelligent and Optimization techniques based on evolutionary computing, metaheuristic,biological base,nature inspired, search method establish their applications in the area of electrical economic power dispatch planning(EEPDP) to reach global optimal solution for this multi scale, multi-decision, multi-objective combinatorial problem subjected to different constraints.An application of ALO to solve non linear electric economic power dispatch problem(EEPDP) is proposed in this paper. Efficient and optimal planning of economic electrical power dispatch problem is an integral part of economic electrical energy generation planning and it is the need of time for the electrical engineers to browse this area in multi-scale planning scenarios. The performance of $s$ ant lion optimizer (ALO) to solve electrical economic power dispatch problem is tested on three and six unit system.Test results are compared with other techniques grey wolf optimization(GWO),cuckoo search(CS), artificial bee colony $(A B C)$,firefly algorithm( $F A)$,particle swarm optimization(PSO), shuffled frog leap $(S F L)$, bacteria foraging algorithm(BFO), harmony search(HS) applied in literature. Simulation results proved that the ALO technique is better as compared to other nature inspired,heuristic,metaheuristic techniques to find global minima and maintain the solution quality in terms of low fuel cost.
\end{abstract}

Keywords: Ant Lion Optimizer (ALO), Electrical Economic Power Dispatch Problem (EEPDP), artificial bee colony $(A B C)$,firefly algorithm $(F A)$,particle swarm optimization(PSO), shuffled frog leap (SFL)

\section{Introduction}

The EEPDP is an imperative area of today's power system planning. The purpose of the EEPDP is to find the optimum generation among the existing units, such that the total generation cost is minimized while simultaneously satisfying the power balance equations and various other constraints in the system. Shazia Khan[1] presents economic load dispatch (ELD) is the process of scheduling the generating units in such a way to full fill the load demand with satisfied constraints to minimize the total production cost of the thermal power plant. In this paper the Economic load dispatch problem is the Improved version of particle swarm optimization technique where the Particle swarm 
optimization (PSO) is a population- based optimization technique and it is based on the fish schooling. PSO can be applied to a wide range of problems but it lacks the global search ability in the last stage of iterations. This paper used a novel PSO with a inertia weight Improved (IWIPSO), which enhances the ability of particles to explore the solution spaces more effectively and increases their convergence rates. In this paper the power and usefulness of the NWIPSO algorithm is established through its application for $13 \& 15$ generator systems with constraints. AlRashidi, M.R.[2] presents Particle swarm optimization (PSO) has received increased attention in many research fields recently. This paper presents a comprehensive coverage of different PSO applications in solving optimization problems in the area of electric power systems. It highlights the PSO key features and advantages over other various optimization algorithms. Furthermore, recent trends with regard to PSO development in this area are explored. This paper also discusses PSO possible future applications in the area of electric power systems and its potential theoretical studies. Payal Mistry and Sanjay Vyas[3] presents a successful adaptation of the particle swarm optimization (PSO) algorithm to solve types of economic dispatch (ED) problems in power systems. Economic load dispatch is a non linear optimization problem which is of great importance in power systems. Economic load dispatch is the scheduling of generators to minimize the total operating cost depending on equality and inequality constraints .The transmission line loss has been kept as minimum as possible. The study is carried out for three unit test system for without loss and with loss cases. Shubham Tiwari [4] presents Economic load dispatch is a non linear optimization problem which is of great importance in power systems. In systematical methods suffer from slow convergence and curse of dimensionality in particle swarm optimization can be an wellorganized alternative to solve on large scale non linear optimization problems. This paper presents an overview of basic PSO to provide a complete survey on the problem of economic load dispatch as an optimization problem. The study is carried out for three unit test system and then for six unit generating system for without loss and with loss cases. Adriane B. S. Serapião[5] presents . Economic Dispatch Problems of electric power system is to schedule the committed generating units outputs so as to meet the necessary load demand at less operating cost. In this paper, two test systems of these problems are solved by using the Cuckoo Search (CS ) Algorithm. A comparison of obtained simulation results by using the CS is carried out against six others swarm intelligence algorithms: Particle Swarm Optimization, Bacterial Foraging Optimization, Artificial Bee Colony, Harmony Search and Firefly Algorithm, Shuffled Frog Leaping Algorithm. The efficiency of each swarm intelligence algorithm is established on a test system comprising three -generators and other containing six-generators. Results denotes the power of the Cuckoo Search Algorithm and confirm its potential to solve the ELD problem. The economic load dispatch is used to define the production cost of each plant, means that the total cost of generation of the system and transmission that is minimum for a prescribed schedule of load. That is to determine production levels of different plants such that the total operating cost is minimum, and at the same time the total load demand and the load losses at any instant are met by the total generation. For an interconnected networks of the system, it is essential to minimize the operating cost. Economic Load Dispatch (ELD) is a process of scheduling the required load demand among the available generating units such that the fuel cost of operating system is minimized. The Economic Load Dispatch problem is formulated as a non-linear constrained optimization problem with both equality and inequality constraints. MM [6] presents Economic load dispatch is a non linear optimization problem which is of great importance in power systems. While analytical methods suffer from slow convergence and curse of dimensionality particle swarm optimization can be an efficient alternative to solve large scale non linear optimization problems. This paper presents an overview of basic PSO to give a comprehensive survey on the problem of economic load dispatch as an optimization 
problem. The study is carried out for three unit test system and then for six unit generating system for without loss and with loss cases.H Abdi [7] presents optimization is one of the very important aspects in power system study fields, especially in power systems, and a verity of models and techniques has been presented in this area. Each model tries to get the best solution as an optimal point, without trapping in local optima. As a powerful tool, modeling based on expert systems and simulation of normal process has an noticeable value in this regard. Since most of power system optimization problems are complex and non-linear with satisfied equality and inequality constraints, the heuristic optimization techniques such as Particle Swarm Optimization (PSO) are considered as realistic and powerful solution schemes to obtain the optimal or quasi-optimal solutions in power system optimization problems. As a lots of paper and researches have been published based on applying PSO in power system operation studies, in this paper a general view on PSO proposed models and approaches in this related is presented. The studies such as UC, ED, OPF, Reactive power compensation, Load forecasting, FACTS, and so on based on PSO proposed models, comparing their benefits and drawbacks are presented. V.Karthikeyan [8] presents Economic Dispatch is very important optimization task in the power system. It is the process of allocating generation among the committed units such that the constraints imposed are satisfied and the energy requirements are minimized. More just, the soft computing method has received supplementary concentration and was used in a quantity of successful and sensible applications. Here, an attempt has been made to find out the minimum cost by using Particle Swarm Optimization (PSO) Algorithm using the data of three generating units. In this work, data has been taken such as the loss coefficients with the max-min power limits and cost function. PSO and Simulated Annealing (SA) are applied to find out the minimum cost for different power demand. When the results are compared with the traditional technique, PSO seems to give a better result with better convergence characteristic. All the methods are executed in MATLAB environment. The effectiveness and feasibility of the proposed method were demonstrated economic dispatch problems .Huynh Thi Thanh Binh[9] presents that the Multi-Area Economic Dispatch problem (MAEDP) in deregulated power system environment for practical multi-area cases with tie line constraints. Our objective is to generate allocation to the power generators in such a manner that the total fuel cost is minimized while all operating constraints are satisfied. This problem is NP-hard. In this paper, we propose Hybrid Particle Swarm Optimization (HGAPSO) to solve MAEDP. The experimental results are reported to show the efficiency of proposed algorithms compared to Particle Swarm Optimization with Time-Varying Acceleration Coefficients (PSO-TVAC) and RCGA. D.N. Jeyakumar [10]presents this paper describes a successful adaptation of the particle swarm optimisation (PSO) algorithm to solve various types of economic dispatch (ED) problems in power systems such as, multi-area ED with tie line limits, ED with multiple fuel options, combined environmental economic dispatch, and the ED of generators with prohibited operating zones. Numerical examples typical to each type are solved on Matlab 6.5 platform, using both the PSO method and the classical evolutionary programming (CEP) approach. The results obtained show that the proposed PSO based ED algorithm can provide comparable dispatch solutions with reduced computation time for all types of ED problem.Hardiansyah [11] presents economic load dispatch (ELD) problem is a common task in the analytical planning of a power system, which requires to be optimized. This paper presents an effective and reliable particle swarm optimization (PSO) technique for the economic load dispatch problem. The results have been demonstrated or ELD of standard 3-generator and 6-generator systems with and without consideration of transmission losses. The final results obtained using PSO are compared with conventional quadratic programming and found to be encouraging. Leandro dos [12] 
presents the objective of the Economic Load Dispatch as the process of scheduling the generating units, so that the system load is supplied entirely and most economically while satisfying all units and system equality and equality constraints.

\section{Problem Formulation}

The EEPDP problem may be expressed by minimizing the fuel cost of generator units under constraints. Depending on load variations, the output of generators has to be changed to meet the balance between loads and generation of a power system. The power system model consists of $n$ generating units already connected to the system [34-50].

The EEPDP problem can be expressed as.

$$
\begin{aligned}
& \text { A. Fuel Cost Model } \\
& C(P G i)=\left(a i^{*} P G i^{2}+b i * P G i+c i\right) R s \\
& \mathrm{i}=1 \ldots . \mathrm{N} \\
& \text { where a,b,c are fuel coefficients, } \mathrm{PG} \text { is power generation in MW,C is fuel cost }
\end{aligned}
$$

\section{B. Constraints}

$$
P G i-P D-P L=O
$$

Where $\mathrm{PG}$ is power Generation, $\mathrm{PD}$ power demand ,PL power loss.

$$
P G i, \text { min } \leq P G i \leq P G i, \text { max }
$$

where $\mathrm{i}=1,2 \ldots \ldots \mathrm{N}$ (Power Limits),PGi,min is the minimum limit of power generation and $\mathrm{PGi}$,max is the maximum limit of power generation.

C.Transmission Losses

$$
P_{L}=\sum_{i=1}^{N} \sum_{j=1}^{N} P_{G i} B_{i j} G_{j}+\sum_{i=1}^{N} B_{0 i} P_{G i}+B o o
$$

Where Boo, Bij , Boi are transmission loss coefficients

\section{Ant Lion Optimizer}

Ant lion optimizer(ALO) is new meta heuristic algorithm developed by Syedalli[51].This is motivated from the hunting mechanism of ant lions .Inherit steps of hunting prey such as the random walk of ants, building traps, entrapment of ants in traps, catching preys, and re-building are simulated to find the optimal solution of real life problems.

\section{A. Inspiration}

Antlions are from Myrmeleontidae family and Neuroptera order. The lifecycle of antlions includes two main phases: larvae and adult. A natural total life can take up to 3 years, which mostly occurs in larvae. Antlions follow metamorphosis in a cocoon to become adult. They mostly hunt in larvae and the adulthood period is for reproduction. Their names originate from their unique hunting behaviour and their favourite prey. An antlion larvae digs a cone-shaped pit in sand by moving along a circular path and throwing out sands with its massive jaw. After digging the trap, the larvae hides underneath the bottom of the cone and waits for insects to be trapped in the pit. The edge of the cone is sharp enough for insects to fall to the bottom of the trap easily. Once the antlion realizes that a prey is in the trap, it tries to catch it. However, insects usually are not caught immediately and try to escape from the trap. In this case, antlions intelligently throw sands towards to edge of the pit to slide the prey into the bottom of the pit. When a prey is caught into the jaw, it is pulled under the soil and consumed. After consuming the prey, antlions throw the leftovers outside the pit and amend the pit for the next hunt. Another interesting behaviour that has been observed in life style of antlions is the relevancy of the size of the 
trap and two things: level of hunger and shape of the moon. Antlions tend to dig out larger traps as they become more hungry and/or when the moon is full. They have been evolved and adapted this way to improve their chance of survival. It also has been discovered that an antlion does not directly observe the shape of the moon to decide about the size of the trap, but it has an internal lunar clock to make such decisions. The main inspiration of the ALO algorithm comes from the foraging behaviour of antlion's larvae.

B. Operators of the ALO algorithm

The ALO algorithm mimics interaction between antlions and ants in the trap. To model such interactions, ants are required to move over the search space, and antlions are allowed to hunt them and become fitter using traps. Since ants move stochastically in nature when searching for food, a random walk is chosen for modeling ants' movement as follows:

$X(t)=|0 ; \operatorname{cumsum}(2 r(t 1)-1) ; \operatorname{cumsum}(2 r(t 2)-1) ; \ldots ; \operatorname{cumsum}(2 r(t n)-1)|$

where cumsum calculates the cumulative sum, $\mathrm{n}$ is the maximum number of iteration, $\mathrm{t}$ shows the step of random walk (iteration in this study), and $r(t)$ is a stochastic function defined as follows:

$$
\begin{array}{r}
r(t)=\mid 1 \text { if rand }>0.5 \mid \\
\mid 0 \text { if rand } \leq 0.5 \mid
\end{array}
$$

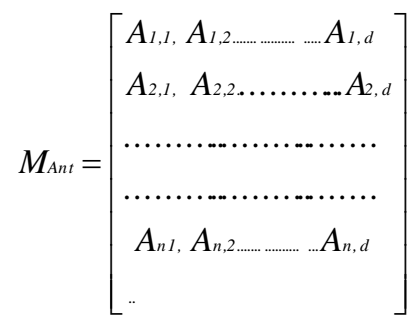

where $\mathrm{M}_{\mathrm{Ant}}$ is the matrix for saving the position of each ant, $\mathrm{A}_{\mathrm{i}, \mathrm{j}}$ shows the value of the $j$-th variable (dimension) of $i_{\text {th }}$ ant, $n$ is the number of ants, and $d$ is the number of variables. The position of an ant refers the parameters for a particular solution. Matrix $\mathbf{M}_{\mathrm{Ant}}$ has been considered to save the position of all ants (variables of all solutions) during optimization.

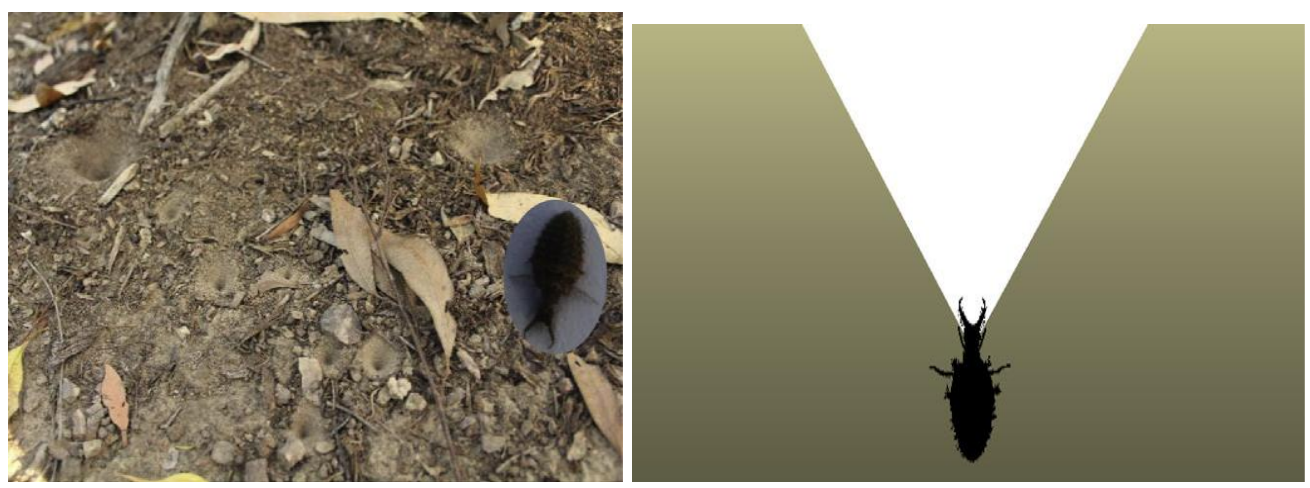

Figure 1. Cone Shape Trap and Hunting Behavior of Ants 
For evaluating each ant, a fitness function is used during optimization and following matrix stores the fitness value of all ants:

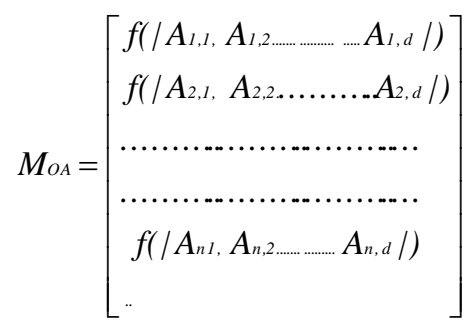

where $\mathrm{M}_{\mathrm{OA}}$ is the matrix for saving the fitness of each ant, $A_{i, j}$ shows the value of $j^{\text {th }}$ dimension of $\mathrm{i}_{\mathrm{th}}$ ant, $\mathrm{n}$ is the number of ants, and $\mathrm{f}$ is the objective function. In addition to ants, we assume the antlions are also hiding somewhere in the search space. In order save their positions and fitness values, the following matrices are utilized:

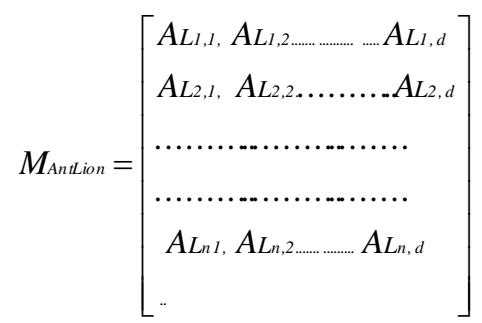

where $M_{\text {Antlion }}$ is the matrix for saving the position of each antlion, $A L_{i, j}$ shows the $j^{\text {th }}$ dimension's value of $i^{\text {th }}$ antlion, $n$ is the number of antlions, and $d$ is the number of variables.

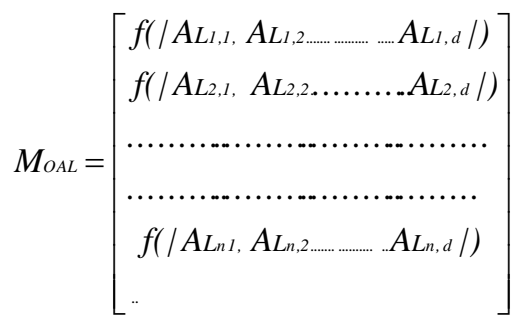

where $\mathrm{M}_{\mathrm{OAL}}$ is the matrix for saving the fitness of each antlion, $\mathrm{AL}_{\mathrm{i}, \mathrm{j}}$ shows the $\mathrm{j}^{\text {th }}$ dimension's value of $i^{\text {th }}$ antlion, $n$ is the number of antlions, and $f$ is the objective function.

During optimization, the following conditions are applied:

- Ants move around the search space using different random walks.

- Random walks are applied to all the dimension of ants.

- Random walks are affected by the traps of antlions.

- Antlions can build pits proportional to their fitness (the highermfitness, the larger pit).

- Antlions with larger pits have the higher probability to catch ants.

- Each ant can be caught by an antlion in each iteration and themelite (fittest antlion).

- The range of random walk is decreased adaptively to simulate sliding ants towards antlions.

- If an ant becomes fitter than an antlion, this means that it is caught and pulled under the sand by the antlion.

- An antlion repositions itself to the latest caught prey and builds a pit to improve its change of catching another prey after each hunt. 


\section{Random walks of ants}

Ants update their positions with random walk at every step of optimization. Since every search space has a boundary (range of variable). In order to keep the random walks inside the search space, they are normalized using the following equation (minmax..normalization):

$x_{i}^{t}=\left(x_{i}^{t}-a_{i}\right) \times\left(d_{i}-c_{i}^{t}\right) /\left(d_{i}^{t}-a_{i}\right)+c_{i}$

where $a_{i}$ is the minimum of random walk of $i_{t h}$ variable, $b_{i}$ is the maximum of random walk in $\mathrm{i}_{\mathrm{th}}$ variable, $\mathrm{c}_{\mathrm{i}}^{\mathrm{t}}$ is the minimum of $\mathrm{i}^{\text {th }}$ variable at $\mathrm{t}^{\text {th }}$ iteration, and $\mathrm{d}_{\mathrm{i}}^{\mathrm{t}}$ indicates the maximum of $\mathrm{i}^{\text {th }}$ variable at $\mathrm{t}^{\text {th }}$ iteration. Eq. (11) should be applied in each iteration to guarantee the occurrence of random walks inside the search space.

D. Trapping in antlion's pits

As discussed above, random walks of ants are affected by antlions' traps. In order to mathematically model this assumption, the following equations are proposed:

$c_{i}^{t}=\operatorname{Antlion}_{j}^{t}+c^{t}$

$d_{i}^{t}=\operatorname{Antlion}_{j}^{t}+d^{t}$

where $\mathrm{c}^{\mathrm{t}}$ is the minimum of all variables at $\mathrm{t}^{\text {th }}$ iteration, $\mathrm{d}^{\mathrm{t}}$ indicates the vector incorporating the maximum of all variables at $t^{\text {th }}$ iteration, $c^{t}{ }_{j}$ is the minimum of all variables for $i^{\text {th }}$ ant, $\mathrm{d}^{\mathrm{t}} \mathrm{j}$ is the maximum of all variables for $\mathrm{i}^{\text {th }}$ ant, and Antliont $\mathrm{j}$ shows the position of the selected $j^{\text {th }}$ antlion at $t^{\text {th }}$ iteration. Eqs. (12) and (13) show that ants randomly walk in a hyper sphere defined by the vectors $\mathrm{c}$ and $\mathrm{d}$ around a selected antlion.

\section{E. Building trap}

In order to model the antlions's hunting capability, a roulette wheel is employed.Ants are assumed to be trapped in only one selected antlion. The ALO algorithm is required to utilize a roulette wheel operator for selecting antlions based of their fitness during optimization. This mechanism gives high chances to the fitter antlions for catching ants.

\section{F. Sliding ants towards antlion}

With the mechanisms proposed so far, antlions are able to build traps proportional to their fitness and ants are required to move randomly. However, antlions shoot sands outwards the center of the pit once they realize that an ant is in the trap. This behavior slides down the trapped ant that is trying to escape. For mathematically modelling this behaviour, the radius of ants's random walks hyper-sphere is decreased adaptively. The following equations are proposed in this regard:

$$
\begin{aligned}
& \mathrm{c}^{\mathrm{t}}=\mathrm{c}^{\mathrm{t}} / I \\
& \mathrm{~d}^{\mathrm{t}}=\mathrm{d}^{\mathrm{t}} / I
\end{aligned}
$$

where $I$ is a ratio, $c^{t}$ is the minimum of all variables at $t^{\text {th }}$ iteration, and $d^{t}$ indicates the vector including the maximum of all variables at $t^{\text {th }}$ iteration. $T$ is the maximum number of iterations, and $w$ is a constant defined based on the current iteration $(w=2$ when $t>$ $0.1 \mathrm{~T}, \mathrm{w}=3$ when $\mathrm{t}>0.5 \mathrm{~T}, \mathrm{w}=4$ when $\mathrm{t}>0.75 \mathrm{~T}, \mathrm{w}=5$ when $\mathrm{t}>0.9 \mathrm{~T}$, and $\mathrm{w}=6$ when $\mathrm{t}$ $>0.95 \mathrm{~T})$. Basically, the constant $\mathrm{w}$ can adjust the accuracy level of exploitation. 


\section{G. Catching prey and re-building the pit}

The final stage of hunt is when an ant reaches the bottom of the pit and is caught in the antlion's jaw. After this stage, the antlion pulls the ant inside the sand and consumes its body. For mimicking this process, it is assumed that catching prey occur when ants becomes fitter (goes inside sand) than its corresponding antlion. An antlion is then required to update its position to the latest position of the hunted ant to enhance its chance of catching new prey.

The following equation is proposed in this regard:

$$
\operatorname{AntLion}_{\mathrm{j}}^{\mathrm{t}}=\text { Ant }_{\mathrm{i}}^{\mathrm{t}} \text { if } \text { Ant }_{\mathrm{i}}^{\mathrm{t}}>f\left(\text { AntLion }_{\mathrm{j}}^{\mathrm{t}}\right)
$$

where $t$ shows the current iteration, Antlion ${ }_{j}{ }_{j}$ shows the position of selected $\mathrm{j}^{\text {th }}$ antlion at $\mathrm{t}^{\text {th }}$ iteration, and $\mathrm{Ant}_{\mathrm{i}}^{\mathrm{t}}$ indicates the position of $\mathrm{i}^{\text {th }}$ ant at $\mathrm{t}^{\text {th }}$ iteration.

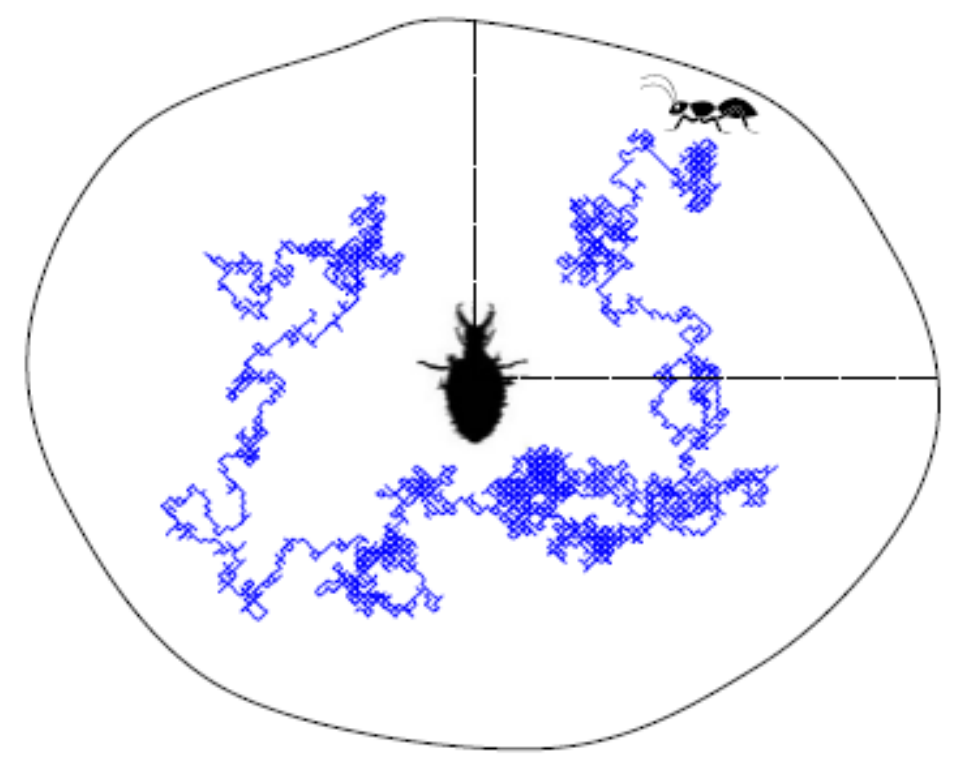

Figure 2. Random Walk of Ant Inside Ant Trap [51]

\section{H. Elitism}

Elitism is an important characteristic of evolutionary algorithms that allows them to maintain the best solution(s) obtained at any stage of optimization process. In this study the best antlion

obtained so far in each iteration is saved and considered as an elite. Since the elite is the fittest antlion, it should be able to affect the movements of all the ants during iterations. Therefore, it is assumed that every ant randomly walks around a selected antlion by the roulette wheel and the elite simultaneously as follows:

$$
\mathrm{Ant}_{\mathrm{i}}^{\mathrm{t}}=R_{A}^{t}+R_{E}^{t} / 2
$$

where $\mathrm{R}_{\mathrm{A}}^{\mathrm{t}}$ is the random walk around the antlion selected by the roulette wheel at $\mathrm{t}^{\text {th }}$ iteration, $R_{E}^{t}$ is the random walk around the elite at $t^{\text {th }}$ iteration, and Ant ${ }^{t} i$ indicates the position of $\mathrm{i}$-th ant at $\mathrm{t}$-th iteration. 


\section{ALO Algorithm}

With the proposed operators in the preceding subsections, the ALO optimization algorithm can now be defined. The ALO algorithm is defined as a three-tuple function that approximates the global optimum for optimization problems as follows:

where $\mathrm{A}$ is a function that generates the random initial solutions, $\mathrm{B}$ manipulates the initial population provided by the function $\mathrm{A}$, and $\mathrm{C}$ returns true when the end criterion is satisfied. The functions A, B and C are defined as follows:

$$
\begin{gathered}
\theta \rightarrow^{A}\left\{M_{\text {Ann }}, M_{O A}, M_{\text {Antlion }}, M_{O A L}\right\} \\
\left\{M_{\text {Ant }}, M_{\text {Antion }}\right\} \theta \rightarrow^{B}\left\{M_{\text {Ant }}, M_{\text {Antlion }}\right\} \\
\left\{M_{\text {Ant }}, M_{\text {Antion }}\right\} \theta \rightarrow^{C}\{\text { True, False }\}
\end{gathered}
$$

where $\mathbf{M}_{\text {Ant }}$ is the matrix of the position of ants, $\mathbf{M}_{\text {Antlion }}$ includes the position of antlions, $\mathrm{M}_{\mathrm{OA}}$ contains the corresponding fitness of ants, and $\mathrm{M}_{\mathrm{OAL}}$ has the fitness of antlions.

\section{J. Pseudo Code}

Initialize the first population of ants and antlions randomly

Calculate the fitness of ants and antlions

Find the best antlions and assume it as the elite

while the end criterion is not satisfied for every ant

Select an antlion using Roulette wheel

Update $\mathrm{c}$ and $\mathrm{d}$

Create a random walk and normalize it

Update the position of ant

end for

Calculate the fitness of all ants

Replace an antlion with its corresponding ant it if becomes fitter

Update elite if an antlion becomes fitter than the elite

end while

Return elite

\section{K. Exploration}

In the ALO algorithm, the antlion and ant matrices are initialized randomly using the function A. In every iteration, the function B updates the position of each ant with respect to an antlion

selected by the roulette wheel operator and the elite. The boundary of position updating is first defined proportional to the current number of iteration. The updating position is then accomplished by two random walks around the selected antlion and elite. When all the ants randomly walk, they are evaluated by the fitness function. If any of the ants become fitter than any other antlions, their positions are considered as the new positions for the antlions in the next iteration. The best antlion is compared to the best antlion found during optimization (elite) and substituted if it is necessary. These steps iterative until the function $\mathrm{C}$ returns false.

Theoretically speaking, the proposed ALO algorithm is able to approximate the global optimum of optimization problems due to the following reasons:

- Exploration of the search space is guaranteed by the random selection of antlions and random walks of ants around them. 
- Exploitation of search space is guaranteed by the adaptive shrinking boundaries of antlions' traps.

- There is high probability of resolving local optima stagnation due to the use of random walks and the roulette wheel.

- ALO is a population-based algorithm, so local optima avoidance is intrinsically high.

- Intensity of ants' movement is adaptively decreased over the course of iterations, which guarantees convergence of the ALO algorithm.

- Calculating random walks for every ant and every dimension promotes diversity in the population.

- Antlions relocate to the position of best ants during optimization, so promising areas of search spaces are saved.

- Antlions guide ants towards promising regions of the search space.

- The best antlion in each iteration is saved and compared to the best antlion obtained so far (elite).

- The ALO algorithm has very few parameters to adjust.

- The ALO algorithm is a gradient-free algorithm and considers problem as a black box.

\section{Optimal Economic Power Dispatch Formulation using ALO}

\section{The Variables[34-50]}

Power Generation (PG) and cost coefficients $(a, b, c)$ of units with objective function as fuel cost, quadratic in nature. Power Generation variable should be initialized as starting point for initial solution in ALO [51] where A is power generation values.

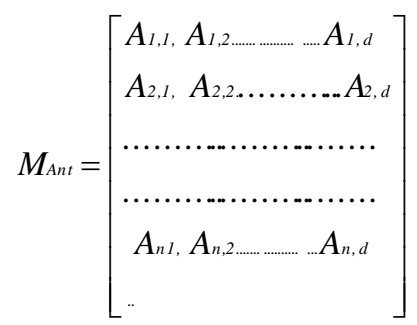

\section{Constraints[34-50]}

Equality Constraints: $\left(\mathrm{P}_{\mathrm{G}}-\mathrm{P}_{\mathrm{D}}-\mathrm{P}_{\mathrm{L}}=0\right)$

In-Equality Constraints: Power Generation should be between minimum and maximum limit of power generation.

Variables in constraints should be incorporated in ALO algorithm.

$>$ Stopping Criteria

It is maximum iteration limit for optimum solution.

\section{Test System}

This case study incorporates three and six generating units. The coefficients of fuel cost and the limits of the generation units are given in Table 1 and 2 .

$$
\text { Boo }=0.056, \text { Bio }=0.001 \times[-0.3908,-0.1297,0.7047,0.0591,0.2161,-0.6635]
$$

This case study incorporates three generating units. The coefficients of fuel cost and the capacities of the generation units are mentioned in Table 1. 
Table 1. Generator Data for Test System III(Load Demand-150 MW)

\begin{tabular}{|l|c|c|c|c|c|}
\hline Unit & $\mathrm{a}(\$ / \mathrm{MW} 2)$ & $\mathrm{b}(\$ / \mathrm{MW})$ & $\mathrm{c}(\$)$ & $\mathrm{PG}_{\min }(\mathrm{MW})$ & $\mathrm{PG}_{\max }(\mathrm{MW})$ \\
\hline 1 & 0.008 & 7 & 200 & 10 & 85 \\
\hline 2 & 0.009 & 6.3 & 180 & 10 & 80 \\
\hline 3 & 0.007 & 6.8 & 140 & 10 & 70 \\
\hline
\end{tabular}

This case study incorporates three generating units. The coefficients of fuel cost and the limits of the generation units are given in Table 1 .

$$
\begin{aligned}
& \text { Boo }=0.030523, \text { Bio }=0.001 \times[0.3,3.1,1.5] \\
& B i j=0.01 \times[0.0218,0.1193,0.0028 ; 0.0093,0.0228,0.0017 ; 0.0028,0.0017,0.0179]
\end{aligned}
$$

Where Boo,Bij,Bio are transmission loss coefficients

Table 2. Generator Data for Test System II (Load Demand - 700 MW)

\begin{tabular}{|l|c|c|c|c|c|}
\hline Unit & $\mathrm{a}(\$ / \mathrm{MW} 2)$ & $\mathrm{b}(\$ / \mathrm{MW})$ & $\mathrm{c}(\$)$ & $\mathrm{PG}_{\min }(\mathrm{MW})$ & $\mathrm{PG}_{\max }(\mathrm{MW})$ \\
\hline 1 & 0.007 & 7 & 240 & 100 & 500 \\
\hline 2 & 0.005 & 10 & 200 & 50 & 200 \\
\hline 3 & 0.009 & 8.5 & 220 & 80 & 300 \\
\hline 4 & 0.009 & 11 & 200 & 50 & 150 \\
\hline 5 & 0.0080 & 10.5 & 220 & 50 & 200 \\
\hline 6 & 0.0075 & 12 & 120 & 50 & 120 \\
\hline
\end{tabular}

$$
B i j=0.001 \times\left[\begin{array}{l}
0.14,0.17,0.15,0.19,0.26,0.22 ; \\
0.17,0.6,013,0.16,015,0.2 ; \\
0.15,0.13,0.65,0.17,0.24,0.19 \\
0.19,0.16,0.17,0.71,0.3,0.25 ; \\
0.26,0.15,0.24,0.3,069,0.32 ; \\
0.22,0.2,019,0.25,032,0.85
\end{array}\right]
$$

Where Bio,Boo,Bij are loss coefficients

\section{Simulation Results}

Table 3. Results Comparison with other Techniques on Three Unit System [44]

\begin{tabular}{|l|l|l|l|l|}
\hline Parameters & ALO & CS[44] & ABC[44] & FA[44] \\
\hline $\begin{array}{l}\text { PG1 } \\
\text { (MW) }\end{array}$ & $\mathbf{3 8 . 1 6}$ & 33.490 & 33.049 & 32.729 \\
\hline $\begin{array}{l}\text { PG2 } \\
\text { (MW) }\end{array}$ & $\mathbf{7 0 . 8 2}$ & 64.116 & 61.764 & 63.843 \\
\hline $\begin{array}{l}\text { PG3 } \\
\text { (MW) }\end{array}$ & $\mathbf{4 3 . 0 0}$ & 55.126 & 57.872 & 56.151 \\
\hline $\begin{array}{l}\text { Cost } \\
(\$ / h r)\end{array}$ & $\mathbf{1 5 9 5 . 5 6}$ & 1600.46 & 1600.51 & 1600.47 \\
\hline
\end{tabular}

In table 3, result comparison has been shown of ALO algorithm with other algorithms firefly algorithm(FA), cuckoo search(CS),artificial bee colony(ABC) in literature. Power 
generation of each units and total fuel cost is compared. From results we can see that proposed method results in less fuel cost as compared to other algorithms.

\section{Table 4.Results Comparison with other Techniques on Three Unit System [44]}

\begin{tabular}{|l|c|}
\hline Technique & Cost $(\$ / \mathrm{hr})$ \\
\hline ALO & $\mathbf{1 5 9 5 . 5 6}$ \\
\hline CS[44] & 1600.46 \\
\hline ABC[44] & 1600.51 \\
\hline FA[44] & 1600.47 \\
\hline PSO[52] & 1600.60 \\
\hline SFL[52] & 1600.67 \\
\hline BFO[52] & 1600.02 \\
\hline HS[52] & 1600.58 \\
\hline
\end{tabular}

In table 4 , result comparison has been shown of ALO algorithm with other algorithms in literature. Power generation of each units and total fuel cost is compared. From results we can see that proposed method gives competetive results as compared to other algorithms in literature.

Table 5. Results Comparison with other Techniques on Six Unit System (Power Demand-700 MW) [12]

\begin{tabular}{|l|l|l|c|c|c|}
\hline Parameters & ALO & GWO[44] & CS[44] & ABC[44] & FA[44] \\
\hline $\begin{array}{l}\text { PG1 } \\
\text { (MW) }\end{array}$ & $\mathbf{3 4 0 . 9 2 8 1}$ & 272.2641 & 324.113 & 323.043 & 293.312 \\
\hline $\begin{array}{l}\text { PG2 } \\
\text { (MW) }\end{array}$ & $\mathbf{7 1 . 1 5 8 8 6}$ & 85.45712 & 76.859 & 54.965 & 79.546 \\
\hline $\begin{array}{l}\text { PG3 } \\
\text { MW) }\end{array}$ & $\mathbf{8 0}$ & 168.5936 & 158.094 & 147.354 & 123.334 \\
\hline $\begin{array}{l}\text { PG4 } \\
(\text { MW })\end{array}$ & $\mathbf{9 3 . 2 6 7 1 5}$ & 60.89443 & 50.000 & 50.000 & 69.700 \\
\hline $\begin{array}{l}\text { PG5 } \\
(\text { MW })\end{array}$ & $\mathbf{5 0}$ & 73.64845 & 51.963 & 85.815 & 79.546 \\
\hline $\begin{array}{l}\text { PG6 } \\
(M W)\end{array}$ & $\mathbf{7 5 . 6 4 5 8 6}$ & 50.13542 & 50.000 & 50.233 & 63.778 \\
\hline $\begin{array}{l}\text { Cost } \\
(\$ / h r)\end{array}$ & $\mathbf{8 4 6 3 . 9 4}$ & 8352.0153 & 8356.06 & 8372.27 & 8388.45 \\
\hline
\end{tabular}

In table 4 and 5 , result comparison has been shown of ALO algorithm with other algorithms in literature. Power generation of each units and total fuel cost is compared. From results we can see that proposed method gives competetive results as compared to other algorithms in literature.

\section{Conclusion}

An application of ALO in EEPDP has been proposed. Proposed technique is tested on three and six unit system .Test results reflects the minimum operating cost, optimum power generation and high speed convergence of solution. A contrast has been made with other techniques presented in literature. It is superior than other techniques presented in literature in terms of fuel cost and power generation. Hence, ALO algorithm is robust and directed to optimal global solution in EEPDP. 


\section{Acknowledgements}

The authors would like to thank Seyedali Mirjalili, Griffith University for his assistance.

\section{References}

[1] J. John j. Greinger. \& William D. Steveson, Power System Analysis, Singapore, McGraw Hill(1994).

[2] D. K. \&. I. Nagrath, Modern Power System Analysis, New Delhi, Tata Mc Graw Hill(2003).

[3] J. Zhu, Optimization of Power System Operation, Hoboken, New Jersy: John Wiley Son (2009).

[4] S. S. \&. V. V. V. Karthikeyan, A New Approach To The Solution of Economic Load dispatch Using Particle Swarm Optimization with Simulated Annealing, International Journal on Computational Science\& Application, Vol(3): 37-41( 2013).

[5] V. P. R. Amita Mahor, Economic dispatch using particle swarm optimization: A review, Renewable and Sustainable Energy Reviews, India,Vol(2):23-29(2009).

[6] M. M. G. Baskar, "Contingency constrained economic load dispatch," Electric Power Systems Research, Tamil Nadu, India,Vol(3):31-34(2009).

[7] J. Y. M. Hardiansyah, Solving Economic Load Dispatch Problem Using Particle Swarm optimization Technique, I.J. Intelligent Systems and Applications, Vol(3):12-18(2012).

[8] Mohn Faazi, Othman, Rubiyan, Mayzuki Khalid, Solving Economic load Dispatch Using particle Swarm Optimization, Journal of Theoretical \& Applied Information Technology, Vol (46):526-535(2012).

[9] H. M. D. ,. M. P. \&. B. K. P. Kamlesh Kumai Vishwakama, Simulated Annealing For Solving Economic Load Dispatch Problem with Valve Point Loading Dispatch Problem, International Journal of Engineering, Science \& Technology, Vol(4):60-72(2012).

[10] P. M. Sudhakaran, Application of Particle Swarm Optimization for Economic Load Dispatch Problem, The 14th International Conference on Intelligent System Applications to Power Systems, ISAP, pp 4-8(2007).

[11] S. SARANGI, Particle Swarm Optimization Applied To Economic load Dispatch,A THESIS SUBMITTED IN PARTIAL FULFILLMENT OF THE REQUIREMENTS FOR THE DEGREE OF Master of Technology in Power Control and Drives(2009).

[12] K. P. ,Naveen Kumar, A Genetic Algorithm Approach for the Solution of Economic Load Dispatch Problem, International Journal on Computer Science and Engineering, Vol(4):1063-1067(2012).

[13] O. P. M. M. G. Ravinder Singh Maan, Economic Load Dispatch Optimization of Six Interconnected Generating Units Using Particle Swarm Optimization, IOSR Journal of Electrical and Electronics Engineering, $\operatorname{Vol}(6): 21-27$ (2013).

[14] J. S. \&. A. Mohan, Particle Swarm Optimization Approach for Economic Load Dispatch, International Journal of Engineering Research \& Application, Vol(3): 13-22(2013).

[15] S. G. S. P. G. B. Shaw, Solution of Economic Load Dispatch Problems",International Journal on Electrical Engineering and Informatics, Vol(3):26-36(2011).

[16] S. Sarangi, Particle Swarm Optimization Applied To Economic Load Dispatch Problem," International Journal Of Engineering Science Invention,Vol(1):33-39(2009).

[17] N. A. R. ,. A. B. H. M. Mohd Noor Abbullah, Efficient Evolution Particle Swarm Optimization Approach for Non Convex Economic Load Dispatch Problem, International Journal Of Engineering Science Invention, Vol(89):139-143(2013).

[18] O.P.M.M.G. Ravinder Singh Maan, Solution to Economic Load Dispatch Problem with Improved Computational Performance Using Particle Swarm Optimization, International Journal Of Engineering Science Invention, $\operatorname{Vol}(2): 01-06$ (2013) .

[19] .-D.-V. R. I.D.Soubache, Unified Particle Swarm Optimization,International Journal of Innovative Technology and Exploring Engineering, Vol(2):280-283(2013).

[20] A. K. G. C. Shubham Tiwari,Economic Load Dispatch Using Particle Swarm Optimization,International Journal of Application \& Innovation in Engineering \& Management, Vol(2): 2391-4847(2013).

[21] T. Bommirani.B, Optimization Technique for The Economic Dispatch in Power System Operation,International Journal of Computer and Information Technology, Vol(2):158-162(2013).

[22] C.-S. L. Leandro dos Santos Coelho, Solving economic load dispatch problems in power systems using chaotic and Gaussian particle swarm optimization approaches, Electrical Power and Energy Systems, Vol(30):297-307(2008).

[23] Q.Bai, Analysis of Particle Swarm Optimization Algorithm, Journal of Computer \& Information Science, Vol(3):180-182(2010).

[24] S. Talukder, Mathematical Modeling and Applications of Particle Swarm Optimization,Master's Thesis Mathematical Modeling and Simulation(2011).

[25] D. V.Selvi, Comparative Analysis of Ant Colony and Particle Swarm Optimization Techniques, International Journal of Computer Applications, vol(5):1-5(2010).

[26] A. Lazinica, Particle Swarm Optimization, Vienna, Austria: In-Tech(2009). 
[27] Xueming Yang A Modified Particle Swarm Optimization With Dynamic Adaption, Applied Mathematics \& Computation, Vol(8):1205-1213(2007).

[28] T. S. K. B. S. Soodabesh Darzi, Overview of Particle Swarm Optimization on the Application \& Methods,Australian Journal on Basic \&Applied Science, Vol(7):490-499(2013).

[29] Y. K. C. Vinod Puri,A Solution to Economic Dispatch Problem Using Augmented lagrangian Particle Swarm Optimization,International Journal of Emerging Technology and Advanced Engineering, Vol(2): 511- 576(2012).

[30] Yamille del Valle, Particle Swarm Optimization: Basic Concepts, Variants and Applications in Power Systems, IEEE TRANSACTIONS ON EVOLUTIONARY COMPUTATION, Vol(12):33-38(2008).

[31]Y. S. Russel .C. Eberhert,Particle Swarm Optimization",Development, Application \& Resources,Proceedings of IEEE, pp. 81-84(2001).

[32]Y. S. Y. C. G. W. Ying Tan, Economic dispatch using particle swarm optimization ,Advances in Swarm Intelligence, Springer, Vol(2):45-50(2011).

[33] A. Y. Saber, Economic dispatch using particle swarm optimization with bacterial foraging effect, Electrical Power and Energy Systems, Vol(34): 38-46(2012).

[34] Navpreet Singh Tung,Unit Commitment Dynamics-An Introduction, International Journal of Computer Science \& Information Technology Research Excellence, Vol(2):70-74(2012).

[35] Navpreet Singh Tung , Unit Commitment in Power System-A Review, International Journal of Electrical and Power Engineering, Vol(6): 51-57(2012).

[36] Navpreet Singh Tung and Sandeep Chakravorty ,Active Power Dispatch Planning using Differential Evolution, Journal of Industrial and Intelligent Information, Vol(2):200-204(2014).

[37] Navpreet Singh Tung and Sandeep Chakravorty, Active Power Dispatch Planning using Pattern Search, International Journal of Electrical Energy, Vol. 2, No. 3(2014), pp. 216-220.

[38] Vikram Kumar Kamboj, S.K Bath, Mathematical Formulation of Multi Area Unit Commitment Problem, International Journal of Power System Operation and Energy Management , Vol(3):2-8(2013).

[39] Vikram Singh et al, Short Term Unit Commitment and Economic Load Dispatch Problem of thermal Electric Power System Using Particle Swarm Optimization, International Journal of Power System Optimization,Vol(9):56-63 (2014).

[40] Vikram Kumar Kamboj, S.K Bath,Scope of Biogeography Based Optimization for Economic Load Dispatch and Multi-Objective Unit Commitment Problem, International Journal of Energy Optimization and Engineering Vol(3):34-54(2014).

[41] Navpreet Singh Tung, Dr.Sandeep Chakravorty, Neuro Inspired Genetic Hybrid Algorithm for Active Power Dispatch Planning Problem in Small Scale System,International Journal of Hybrid Information Technology, Vol(8):171-184(2015).

[42] Navpreet Singh Tung and Sandeep Chakravorty, Optimized Power Distribution Planning-A Review ,International Journal of Electrical and Electronics Engineering ,Vol(2):53-58(2013) .

[43] Navpreet Singh Tung ,Amit Bhardwaj, , Vikram Kamboj ,Unit Commitment in Power System: A Review,International Journal of Electrical and Power Engineering, Vol(6): 51-57(2012).

[44] Navpreet Singh Tung,Sandeep Chakravorty,Grey Wolf Optimization for Active Power Dispatch Planning Problem Considering Generator Constraints and Valve Point Effect, International Journal of Hybrid Information Technology,Vol(8),117-134(2015).

[45] VK Kamboj, SK Bath, JS Dhillon ,Solution of non-convex economic load dispatch problem using Grey Wolf Optimizer, Neural Computing and Applications,Vol(2):1-16(2015).

[46] VK Kamboj ,A novel hybrid PSO-GWO approach for unit commitment problem, Neural Computing and Applications, $\operatorname{Vol}(2): 1-13(2015)$.

[47] VK Kamboj ,Implementation of hybrid harmony/random search algorithm considering ensemble and pitch violation for unit commitment problem, International Journal of Electrical Power \& Energy Systems, Vol(77):228-249(2015).

[48] VK Kamboj ,Solution of non-convex economic load dispatch problem for small-scale power systems using ant lion optimizer,Neural Computing and Applications, Vol(1):1-12(2015).

[49] VK Kamboj ,A novel hybrid DE-random search approach for unit commitment problem, Neural Computing and Applications, Vol(1): 1-23(2015).

[50] VK Kamboj ,Hybrid HS-random search algorithm considering ensemble and pitch violation for unit commitment problem,Neural Computing and Applications, Vol(1):1-26(2015).

[51] S. Mirjalili, Ant Lion Optimizer, Advances in Engineering Software, 2015.

[52] Adriane B. S. Serapião, Cuckoo Search for Solving Economic Dispatch Load Problem, Intelligent Control and Automation, Vol(4): 385-390(2013). 